\title{
Ultrastructural characteristics of myenteric plexus in patients with colorectal cancer
}

\author{
Agata Zauszkiewicz-Pawlak ${ }^{1}$, Janusz Godlewski² ${ }^{2}$, Przemyslaw Kwiatkowski ${ }^{2}$, \\ Zbigniew Kmiec ${ }^{1,2}$
}

${ }^{1}$ Department of Histology, Medical University of Gdansk, Gdansk, Poland

${ }^{2}$ Department of Human Histology and Embryology, Faculty of Medical Sciences, University of Warmia and Mazury, Olsztyn, Poland

\begin{abstract}
Introduction. It has been found previously that colorectal cancer (CRC) is accompanied by atrophy of myenteric plexuses (MPs) localized close to the tumor. The aim of the study was to compare ultrastructure of MPs localized in the unchanged part of the colon wall distant to CRC tumor with the ultrastructure of MPs in the vicinity of CRC tumor.

Material and methods. The present study was conducted using post-operative material derived from 11 patients with CRC. Samples of colon wall were taken from the margin of cancer invasion and from a macroscopically unchanged segment of the large intestine, immediately fixed and processed according to the standard protocol for transmission electron microscopy studies.

Results. In the MPs localized in the control part of colon wall the presence of numerous unmyelinated axons and cell bodies of neurons, interstitial cells of Cajal and enteroglial cells were observed. As compared to control samples, in the MPs located close to the tumor invasion, expansion of the extracellular matrix and myelin-like structures accompanying some nerve fibers were found. The appearance of mast and plasma cells was observed within MPs in the vicinity of CRC tumor. Sporadically, apoptotic cells were present inside the MPs.

Conclusions. The presence of myelin-like structures and apoptotic cells within MPs located close to tumor invasion suggests that atrophy of MPs may be caused by factors released from CRC tumor.

(Folia Histochemica et Cytobiologica 2017, Vol. 55, No. 1, 6-10)
\end{abstract}

Key words: colorectal carcinoma; enteric nervous system; myenteric plexus atrophy; apoptosis; Cajal cells; electron microscopy

\section{Introduction}

The enteric nervous system (ENS) is basically built up of two ganglionated plexuses, the submucosal plexuses (of Meissner), and the myenteric plexuses (of Auerbach). The submucosal plexuses can be further subdivided into the inner and outer subdivisions, and the intermediate submucosal plexus located between them in some bigger mammals such as pig or human

Correspondence address: A. Zauszkiewicz-Pawlak, M.D., Ph.D. Department of Histology, Medical University of Gdansk Debinki 1 St., 80-211 Gdansk, Poland e-mail: agatapawlak@gumed.edu.pl
[1-3]. Neurons of the above mentioned plexuses produce axons communicating between submucosal and myenteric plexuses into one functional network. The classification of the enteric neurons according to their shape has been pioneered by Dogiel at the end of $19^{\text {th }}$ century and continued later on independently by Furness [4] and Stach [5]. They distinguished up to eight classes of neurons based on cell shape. Apart from neurons, there are several other types of cells accompanying the ENS plexuses in the GI track, such as enteric glial cells (EGCs), connective tissue cells (fibroblasts/fibrocytes, plasmocytes, mast cells), and various classes of interstitial cells [6]. Among these last mentioned cells, using immunolabeling methods, morphologists have described "fibroblast-like cells" 
which are distributed along processes of enteric motor neurons, close to nerve endings and nerve varicosities, and interstitial Cajal cells (ICCs) which accompany nerve fibers [7, 8]. Interstitial cells play important role in the control of the motor activity of the GI tract, as pacemaker cells and mechanoreceptors $[6,8]$. The main function of the EGCs is the structural support for the ENS components. Recent studies showed that the role of EGCs may be more prominent because they form vast communication network via a complex $\mathrm{Ca}^{2+}$-dependent signals that enables EGCs to transmit and integrate information between the cells of the gut microenvironment (neurons, glial cells, connective tissue cells, and smooth muscle cells) [9].

The structure and function of the ENS in functional and inflammatory disorders of the gastrointestinal (GI) tract has been described [10, 11]. However, little is known about the structure of myenteric plexuses in patients with colorectal cancer (CRC). Our group showed that CRC is accompanied by atrophy of galanin-expressing MPs localized close to the tumor invasion as compared to the plexuses localized distantly from CRC tumor $[12,13]$. Also the age-related neurodegenerative disorders like Alzheimer and Parkinson diseases are accompanied by a loss of neurons in myenteric and submucosal plexuses [14]. To determine possible morphological mechanisms of the atrophy of myenteric plexuses located close to the CRC invasion we performed analysis at the ultrastructural level.

\section{Material and methods}

Patients' recruitment. The present study was conducted using post-operative material obtained from 11 patients ( 5 women and 6 men) with $\mathrm{CRC}$ diagnosis. The mean age of the patients was $71 \pm 7.54$ years (mean \pm SEM, range: 53-79 years). Post-operative histopathological analysis confirmed that patients included in this study formed a homogenous group presenting the same degree of adenocarcinoma invasion within the colon wall, defined as T3 by the TNM staging as defined by the American Joint Committee on Cancer (AJCC), moderate degree of adenocarcinoma differentiation grading (G2) and similar anatomical localization of the tumor in sigmoid colon or upper part of rectum. The average size of the tumor was $4.45 \pm 0.5 \mathrm{~cm}$ (mean \pm SEM). Four patients who did not have regional lymph nodes metastasis were classified as the stage II of a cancer, while the other seven patients presented stage III due to lymph node involvement. The tissue samples were collected during surgery at the Department of Oncological Surgery of the Regional Oncological Centre in Olsztyn, Poland. The protocol of this study was approved by the University of Warmia and Mazury Bioethics Commission
(No. 12/2012) and written informed consent was obtained from all patients in the study. All patients had no evidence of obstruction or other disorders of bowel content passage. Moreover, they did not have another serious illness nor were subjected to neo-adjuvant chemo- or/and radiotherapy.

Material collection and transmission electron microscopy. Immediately after surgical resection of the intestine, very thin slices of colon wall sections, less than $1 \mathrm{~mm}$ of thickness, were harvested from the removed part of the large colon. Samples were taken from the margin of cancer invasion (cancer-infiltrated intestinal wall) and from a macroscopically unchanged segment of the colon at a distance of $5-8 \mathrm{~cm}$ from the tumor, as a control tissue. Tissue samples were immediately fixed in $2.5 \%$ glutaraldehyde in $0.1 \mathrm{mM}$ sodium-cacodylate buffer ( $\mathrm{pH}$ 7.4) followed by post-fixation in $2 \%$ osmium tetroxide and dehydration in graded series of ethanol. After infiltration with propylene dioxide:epon mixture and pure epon, sections were embedded to polymerize. Semi-thin and ultra-thin sections (Reichert U3 ultramicrotome, Vienna, Austria) were obtained and finally contrasted using uranyl acetate and lead citrate prior to the examination in JEM 1200EX II transmission electron microscope (Tokyo, Jeol, Japan) at $80 \mathrm{kV}$.

\section{Results}

\section{Ultrastructure of the myenteric plexuses in the un- changed part of colon wall distant to CRC tumor}

The ultrastructural studies revealed normal organization of the myenteric plexus in colon wall distant from CRC invasion (control tissue). Bundles of unmyelinated nerve fibers and perikaryons were located between the circular and longitudinal layers of smooth muscle cells of the muscularis externa (Fig. 1). They were surrounded by extracellular matrix (ECM) containing abundant collagen and less numerous elastic fibers, fibroblasts/fibrocytes and their processes. Nerve fiber bundles (NFBs) were ensheathed by long cytoplasmic processes of the interstitial Cajal cells and fibrocytes, which separated the bundles from surrounding ECM and smooth muscle cells (Fig. 1A). Axonal profiles presented lucent axoplasm, which contained neurofilaments and neurotubules, neurotransmitter vesicles (large granular vesicles with dense core) and occasionally mitochondria (Fig. 1B). Some of the observed axons were embedded in the infoldings of enteric glial cells (Fig. 1C).

\section{Ultrastructure of the myenteric plexuses in the vicinity of CRC tumor}

Generally, the ultrastructure of MPs located close to the CRC invasion was similar to distantly located MPs. Nerve fibers and enteric glial cells embedded in 

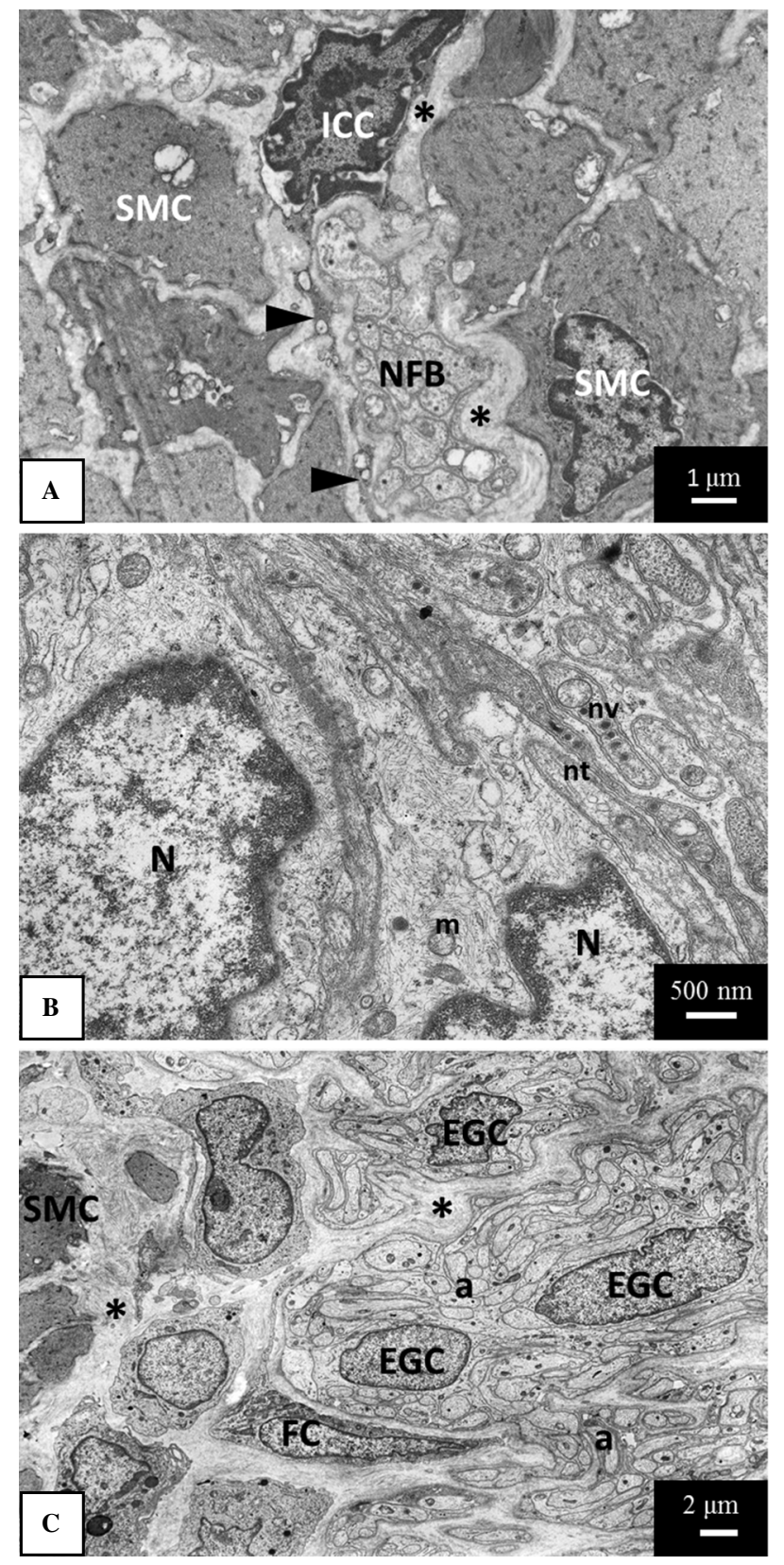

Figure 1. Ultrastructure of myenteric plexuses located distantly from CRC invasion. A. Nerve fiber bundle (NFB) of non-myelinated axons surrounded by smooth muscle cells (SMC). On the top of the bundle interstitial Cajal cell (ICC) with a thin, long protrusion surrounding the bundle from one side (arrowheads). ICC presents irregular, dark, heterochromatic nucleus and scarce cytoplasm. Extracellular matrix (ECM) surrounding the NFB is filled with collagen fibers (asterisk). SMC presents typical phenotype: irregular shape, cytoplasm packed with filaments and dense bodies, attachment plaques are present at the outline. Magnification: $\times 5000$; B. Two neurons (N, nucleus), mitochondria (m) accompanied by axons. Profiles and cross sections of neurotubules (nt) are present inside the axons as well as neurotransmitter vesicles (nv). Magnification: $\times 10000$; C. Enteric glial cells (EGCs) surrounded by numerous axon profiles (a); fibrocyte (FC); smooth muscle cell $(\mathrm{SMC})$. Magnification: $\times 2000$.
ECM rich in collagen fibers between smooth muscle layers (Fig. 2A), being occasionally accompanied by cell bodies and processes of interstitial Cajal cells (Fig. 2B). However, in some of the samples located close to cancer invasion single myelin-like structures within NFBs were present (Fig. 2C). Furthermore, few apoptotic cells were present in the ECM surrounding NFBs (Fig. 2D, 3A). They presented nuclear condensation and shrinkage of the cell body, typical morphological markers of apoptotic cell death. Additionally, we observed increased number of fibroblast-like cells (Fig. 3A) and mast cells (Fig. $3 \mathrm{~B}, \mathrm{C})$ that were present in the ECM surrounding myenteric plexuses. Moreover, ECM was abundant in collagen fibers and seemed to be more prominent than in tissue samples derived from distant region of the colon wall.

\section{Discussion}

Our study presents an attempt to explain the observations by Kwiatkowski et al. [15] and Kozlowska et al. [16] who applied morphometry to measure the area of MPs close to and distant from CRC invasion. They found significant reduction (by approximately $50 \%$ ) of the area occupied by galanin-immunoreactive neurons in the MPs located in the vicinity of CRC invasion in comparison to distally located plexuses. The authors suggested that neither apoptosis nor necrosis has been involved in the observed phenomena $[15,16]$. These finding have been confirmed by the results of our ultrastructural study, which showed neither neurons' apoptosis nor necrosis to occur in myenteric plexuses located close to the CRC tumor. Since the mentioned histomorphometric studies clearly demonstrated atrophy of myenteric plexuses in this location we suggest that the atrophic changes must have taken place earlier during the CRC progression. Since in the present study the patients represented an advanced phase of the disease (stages II and III) it was not possible to determine at the TEM level (this study) or at the light microscopy level, which type of cell death (apoptosis, autophagy or necrosis) was responsible for the atrophy of MPs close to the CRC invasion [16]. Although, we found occasionally presence of apoptotic cells close to the bundles of nerve fibers, the morphology of these cells does not allow defining their type due to cell condensation and shrinkage. Therefore, it cannot be excluded that apoptosis still may play some role in the MPs atrophy in CRC patients. It is also possible that some, as yet unknown factors, released during progression of the disease from the adjacent CRC tumor may cause involution of the neighboring myenteric plexuses. However, this suggestion assumes 

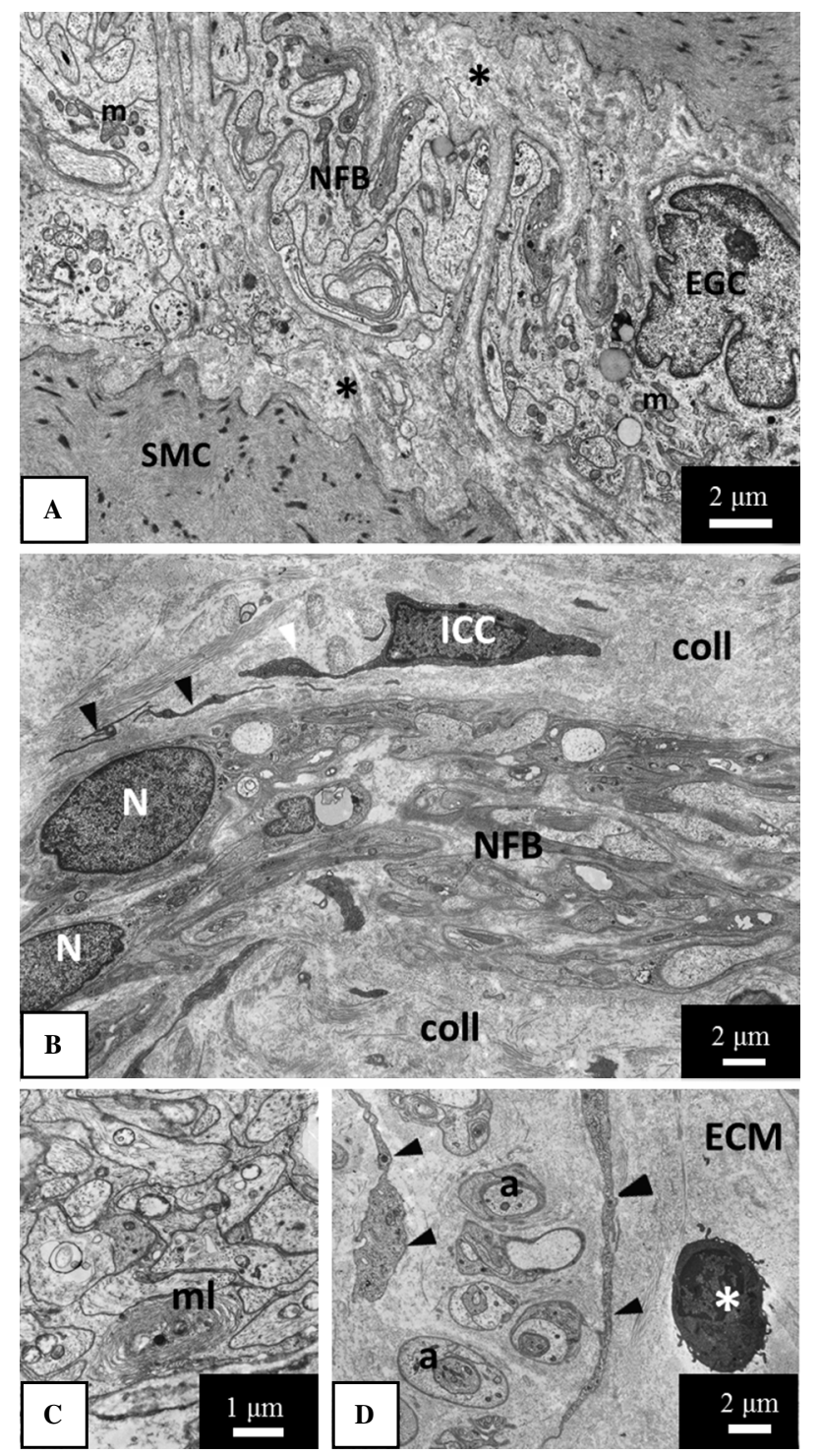

Figure 2. The myenteric plexuses in the vicinity of CRC. A. Enteric glial cell (EGC), mitochondria (m) and a nerve fiber bundle (NFB) surrounded by ECM rich in collagen fibers (asterisk); smooth muscle cell (SMC). Magnification: $\times 2500$; B. Two neurons $(\mathrm{N})$ accompanied by profiles of nerve fibers and a Cajal cell (ICC) producing long, thin protrusions (arrowheads), surrounded by collagen fibers (coll). Magnification: $\times 2000$; C. Myelin-like structure $(\mathrm{ml})$. Magnification: $\times 7500$; D. Axons (a) surrounded by long protrusions of fibroblasts (arrowheads), embedded in ECM. On the right apoptotic cell (asterisk) with condensed nucleus and shrunken cell body. Magnification: $\times 2000$.

analyses of the earlier stages of the disease which was not possible in our study.

The noteworthy finding was the presence of degenerative changes in MPs such as myelin-like structures. Such structures derived from concentrically folded membranes are thought to be associated with neurodegenerative processes $[17,18]$. Although
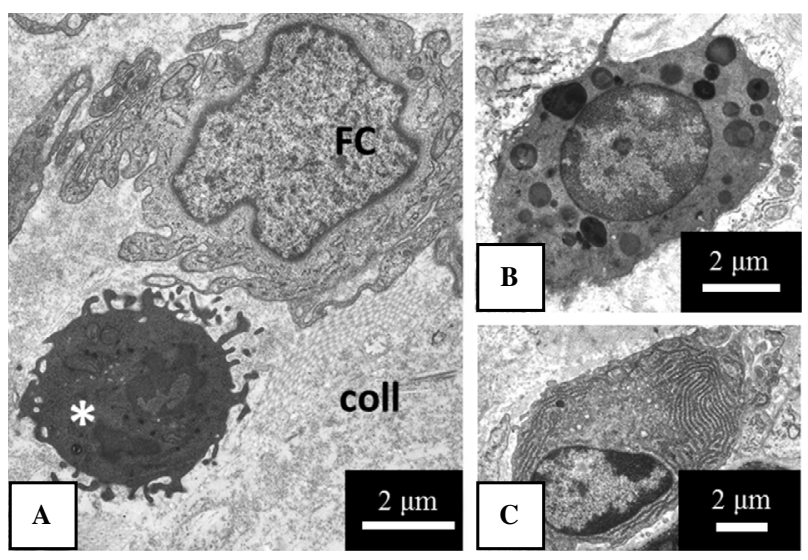

Figure 3. The components adjacent to the myenteric plexus in the vicinity of CRC invasion. A. Fibroblast-like cell (FC) and an apoptotic cell (asterisk) of unknown type with condensed nucleus and shrunken cell body are embedded in extracellular matrix (ECM), rich in collagen fibers (coll). Magnification: $\times 4000$; B. Mast cell. Magnification: $\times 4000$; C. Plasmocyte. Magnification: $\times 2500$.

we observed them mainly close to the CRC invasion, myelin-like bodies were also observed in suprarenal glands and central nervous system [19, 20]. Moreover, the hypertrophied nerve fascicles with prominent perineural sheath which are similar to the extrinsic nerves were demonstrated in Hirschsprung's disease, genetically-determined loss of ENS neurons, in which aganglionic region cause impaired colon peristalsis [21]. Moreover, the increased presence of glial cells and abnormal distribution of collagen fibers and ECM components within colon wall were also reported in Hirschprung's disease [21]. Since we found that samples taken from the margin of cancer invasion were more abundant in collagen fibers and fibroblasts/fibrocytes it may be assumed that these changes may represent the replacement of the atrophied MPs by the connective tissue.

In summary, the presence of myelin-like structures and apoptotic cells within MPs located close to tumor invasion suggests that atrophy of MPs may be caused by factors released from CRC tumor during the progression of the disease.

\section{References}

1. Timmermans JP, Adriaensen D, Cornelissen W, et al. Structural organization and neuropeptide distribution in the mammalian enteric nervous system, with special attention to those components involved in mucosal reflexes. Comp Biochem Physiol A Physiol. 1997; 118(2): 331-340, indexed in Pubmed: 9366065.

2. Timmermans JP, Adriaensen D. Gastrointestinal mechanosensors: analysis of multiple stimuli may require complex sensors. Neurogastroenterol Motil. 2008; 20(1): 4-7, doi: 10.1111/j.1365-2982.2007.01049.x, indexed in Pubmed: 18173558 . 
3. Wedel T, Roblick U, Gleiss J, et al. Organization of the enteric nervous system in the human colon demonstrated by wholemount immunohistochemistry with special reference to the submucous plexus. Ann Anat. 1999; 181(4): 327-337, doi: 10.1016/S0940-9602(99)80122-8, indexed in Pubmed: 10427369

4. Furness JB, Bornstein JC, Trussell DC. Shapes of nerve cells in the myenteric plexus of the guinea-pig small intestine revealed by the intracellular injection of dye. Cell Tissue Res. 1988; 254(3): 561-571, indexed in Pubmed: 3233651.

5. Stach W. A revised morphological classification of neurons in the enteric nervous system. Kluwer Academic Publishers, Lancaster, UK 1989.

6. Furness JB. The enteric nervous system and neurogastroenterology. Nat Rev Gastroenterol Hepatol. 2012; 9(5): 286-294, doi: 10.1038/nrgastro.2012.32, indexed in Pubmed: 22392290.

7. Kurahashi M, Zheng H, Dwyer L, et al. A functional role for the ,fibroblast-like cells' in gastrointestinal smooth muscles. J Physiol. 2011; 589(Pt 3): 697-710, doi: 10.1113/jphysiol.2010.201129, indexed in Pubmed: 21173079.

8. Cobine CA, Hennig GW, Kurahashi M, et al. Relationship between interstitial cells of Cajal, fibroblast-like cells and inhibitory motor nerves in the internal anal sphincter. Cell Tissue Res. 2011; 344(1): 17-30, doi: 10.1007/s00441-0111138-1, indexed in Pubmed: 21337122.

9. Ochoa-Cortes F, Turco F, Linan-Rico A, et al. Enteric glial cells: a new frontier in neurogastroenterology and clinical target for inflammatory bowel diseases. Inflamm Bowel Dis. 2016; 22(2): 433-449, doi: 10.1097/MIB.0000000000000667, indexed in Pubmed: 26689598.

10. Rumessen JJ, Vanderwinden JM, Horn T. Crohn's disease: ultrastructure of interstitial cells in colonic myenteric plexus. Cell Tissue Res. 2011; 344(3): 471-479, doi: 10.1007/s00441-0111175-9, indexed in Pubmed: 21562942.

11. Rumessen JJ, Vanderwinden JM,Horn T, et al. Ulcerative colitis: ultrastructure of interstitial cells in myenteric plexus. Ultrastruct Pathol. 2010; 34(5): 279-287, doi: 10.3109/01913121003770701, indexed in Pubmed: 20568987.

12. Godlewski J, Pidsudko Z. Characteristic of galaninergic components of the enteric nervous system in the cancer invasion of human large intestine. Ann Anat. 2012; 194(4):
368-372, doi: 10.1016/j.aanat.2011.11.009, indexed in Pubmed: 22226150 .

13. Godlewski J. Morphological changes in the enteric nervous system caused by carcinoma of the human large intestine. Folia Histochem Cytobiol. 2010; 48(1): 157-162, doi: 10.2478/v10042010-0029-8, indexed in Pubmed: 20529833.

14. Lebouvier T, Neunlist M, Bruley des Varannes S, et al. Colonic biopsies to assess the neuropathology of Parkinson's disease and its relationship with symptoms. PLoS One. 2010; 5(9): e12728, doi: 10.1371/journal.pone.0012728, indexed in Pubmed: 20856865.

15. Kwiatkowski P, Godlewski J, Kieżun J, et al. Colorectal cancer patients exhibit increased levels of galanin in serum and colon tissues. Oncol Lett. 2016; 12(5): 3323-3329, doi: 10.3892/ol.2016.5037, indexed in Pubmed: 27899999.

16. Kozlowska A, Kwiatkowski P, Oponowicz A, et al. Myenteric plexuses atrophy in the vicinity of colorectal cancer tissue is not caused by apoptosis or necrosis. Folia Histochem Cytobiol. 2016; 54(2): 99-107, doi: 10.5603/FHC.a2016.0012, indexed in Pubmed: 27439439.

17. Saggu SK, Chotaliya HP, Blumbergs PC, et al. Wallerian-like axonal degeneration in the optic nerve after excitotoxic retinal insult: an ultrastructural study. BMC Neurosci. 2010; 11: 97, doi: 10.1186/1471-2202-11-97, indexed in Pubmed: 20707883

18. Schuettauf F, Rejdak R, Walski M, et al. Retinal neurodegeneration in the DBA/2J mouse-a model for ocular hypertension. Acta Neuropathol. 2004; 107(4): 352-358, doi: 10.1007/s00401003-0816-9, indexed in Pubmed: 14745571.

19. Skalska J, Frontczak-Baniewicz M, Strużyńska L. Synaptic degeneration in rat brain after prolonged oral exposure to silver nanoparticles. Neurotoxicology. 2015; 46: 145-154, doi: 10.1016/j.neuro.2014.11.002, indexed in Pubmed: 25447321.

20. Murakoshi M, Osamura Y, Watanabe K. Mitochondrial alterations in aged rat adrenal cortical cells. Tokai J Exp Clin Med. 1985; 10(5): 531-536, indexed in Pubmed: 3837404.

21. Wedel T, Holschneider AM, Krammer HJ. Ultrastructural features of nerve fascicles and basal lamina abnormalities in Hirschsprung's disease. Eur J Pediatr Surg. 1999; 9(2): 75-82, doi: 10.1055/s-2008-1072217, indexed in Pubmed: 10342113.

Submitted: 23 March, 2017

Accepted after reviews: 29 March, 2017

Available as AoP: 31 March, 2017 